\title{
Correction to: Synthesis and Application of a Long-Circulating Radiolabeled Peptide for Targeting of Osteosarcoma
}

Yesen Li, ${ }^{1,2}$ Daifeng Li, ${ }^{2,3}$ Hua Wu, ${ }^{1}$ Jinxiong Huang, ${ }^{1}$ Zhen Cheng ${ }^{3}$

${ }^{1}$ Department of Nuclear Medicine and Minnan PET Center, Cancer Hospital, The First Affiliated Hospital of Xiamen University, Teaching Hospital of Fujian Medical University, Xiamen, China

${ }^{2}$ Department of Orthopedics Trauma and Microsurgery, Zhongnan Hospital of Wuhan University, Wuhan, 430071, Hubei, China

${ }^{3}$ Department of Radiology and Molecular Imaging Program at Stanford, Stanford University School of Medicine, Stanford, CA, 94305, USA

Correction to: Molecular Imaging and Biology

https://doi.org/10.1007/s11307-019-01468-6

This article was updated to correct Figures 1 and 4, which were interchanged. 